\title{
A novel contention probability dynamism for IEEE 802.15.6 standard
}

\author{
Mohammed Fatehy ${ }^{1,2^{*}}$ and Ryuji Kohno ${ }^{1}$
}

\begin{abstract}
The newly emerged IEEE 802.15.6 standard provides a multiple priority-based services for Wireless Body Area Networks (WBAN). Categorizing a node as high/low priority device leads to be assigned a relatively larger/smaller contention probability $(C P)$, respectively. In contention-based protocols, halving the maximum $C P$ value $(C P \max )$ the way down to its minimum value $\left(\mathrm{CP}_{\mathrm{min}}\right)$ has no consideration to the device's awaiting packets in the buffer. Moreover, this halving process shrunken the device's chance to contend to the current time slot which affects the throughput and the average packet service time. In this paper, we propose an amendment to the IEEE 802.15 .6 standard introducing novel CP dynamism based on the device's queue length. The numerical results show an improvement of the proposed dynamism over the conventional in terms of throughput, delay and data packet dropping rate. In addition, the proposed scheme showed a stability in a realistic channel condition.
\end{abstract}

Keywords: Body area network; UWB; Contention; Probability; Medical sensors; IEEE 802.15.6; Amendment

\section{Introduction}

In the IEEE 802.15.6 Wireless Body Area Networks (WBAN) standard [1], an optimization for low-power inbody/on-body nodes is aimed to serve a variety of medical and non-medical applications [2].

The coexistence of many BANs in the near vicinity of each other (elevator for example) can lead to interference between these BANs because of the large number of sensors each piconet can have and unpredictable movement of these sensors. In addition, no proper global coordination scheme exists as there is no natural choice of coordinator between piconets [3]. The previous factors cause a considerable degradation in the performance for each interfering piconet in the near vicinity. Generally, co-channel interference between the different piconets in a WBAN, can be mitigated by using multi-access schemes like the direct sequence ultra wideband (DSUWB) scheme.

Since these nodes are employed for different applications, it imposes the occurrence of different traffic patterns and/or quality of services (QoSs). This leads to the

*Correspondence: hafez-mohammed-tj@ynu.jp

1 Physical, Electrical and Computer Engineering Department, Yokohama National University, 79-1 Tokiwadai, Hodogaya-Ku, 240-8501 Yokohama, Japan

${ }^{2}$ Mathematics Department, Faculty of Science, Suez Canal University, Round Road, 41522 Ismailia, Egypt coexistence of a mixture of these nodes with different requirements in the same WBAN [3]. In other words, the network consists of multi nodes with various priority in this WBAN systems [4]. The IEEE 802.15.6 standard introduced a priority-based scheme in order to fulfill the expected differences in QoS in order to grantee fairness to all nodes.

The new standard defines three physical (PHY) protocols, namely, NarrowBand (NB), Ultra WideBand (UWB), and Human Body Communications (HBC). As Medium Access Control (MAC) protocols, the standard defines also three MAC protocols, namely, scheduled access and scheduled-polling access, improvised access and unscheduled access, and random access which applied either via CSMA/CA or slotted Aloha. We assume in this paper that a system uses UWB over slotted Aloha MAC.

In this paper and for all scenarios, we assume that a coexistence between nodes, belonging to different orientations, try to contend to the same time slot in the contention access period (CAP). In the conventional systems that employ slotted Aloha [5] as its CAP scheme, if two or more packets overlapped, even partially, a collision is considered to have occurred and a retransmission request for all the packets will be issued.

In a previous paper [6], we proposed to employ spreading slotted Aloha scheme in the new IEEE 802.15.6

\section{Springer}

(c) 2014 Fatehy and Kohno; licensee Springer. This is an Open Access article distributed under the terms of the Creative Commons Attribution License (http://creativecommons.org/licenses/by/2.0), which permits unrestricted use, distribution, and reproduction in any medium, provided the original work is properly credited. 
standard as a configurable system applied to multiple priority services. Spreading slotted Aloha has been used previously $[7,8]$ for satellite and other applications but not for WBAN. As another contention access-based MAC protocol, CSMA/CA for IEEE 802.15.6 has been analyzed in different literatures $[9,10]$. During these schemes, the throughput, delay and energy efficiency are the major metrics to evaluate the performance.

The novelty of spreading slotted Aloha is the combination between spreading technique and the slotted Aloha MAC protocol proposed in the standard in order to allow multiple packets to be transmitted and overlapped in the same time slot, without being considered as a collision. The spreading slotted Aloha scheme relays on the characteristics of the spreading techniques to extract each user's packets correctly.

In the previous work, we show the improvement in the probability of correctly retrieving the transmitted packets (throughput) under the proposed spreading slotted Aloha applied for IEEE 802.15.6 standard. We conclude that, in general, the proposed scheme achieved a better throughput comparing to the conventional slotted Aloha proposed in the standard. But the delay resulted due to the spreading code usage is always a tradeoff factor against the achieved throughput in the spreading schemes generally.

As we expected, the spreading code length assigned to the different nodes contending to the medium, plays an important role in the overall system's performance.

Another important observation was that the contention probability $(C P)$ value assigned to every node, according to its priority, has a considerable effect on the system's performance. For nodes with high contention priority (usually medical nodes), it was shown that these nodes achieved much higher throughput combined with much lower delay comparing to the throughput and delay achieved by low priority nodes which employed for entertainment purposes.

Because the entertainment devices usually serve an audio or video streaming applications with continuous traffic, its generated data rate is higher than medical devices which serve a discrete transmission traffic. This combination of different data rate and the different nodes' priorities is another reason for the results we get in the previous work.

Moreover, the initial $\mathrm{CP}$ value (the maximum value, $\mathrm{CP}_{\max }$ according to the current standard) assigned to the low priority nodes is relatively small which increases the period as each packet stays in the system in order to be transmitted.

Finally, the reason we focus on in this paper is the dynamism of lowering the $\mathrm{CP}$ value in case of failure in transmission. The current dynamism is going as halving the $\mathrm{CP}$ value every two transmission failures which adds more delay to the packet in process and to the total delay of the node especially if the node's data generation rate is high which increases the node's queue length or increase the overflow dropped packets.

For the previous reasons, we propose a novel modification for the dynamism of lowering CP which takes into account the node's current queue length as an effective factor for choosing the next $\mathrm{CP}$ value in case of failure to transmit the current packet.

The remaining sections of this paper is organized as follow: Section 2 discuss some previously related research. In Section 3, we give a brief description of the current dynamism of changing the CP value in the IEEE 802.15.6 standard and introduce and state the proposed dynamism. Simulation scenario, parameters and numerical results are presented in Section 4. The conclusion and open problem is drawn in Section 5.

\section{Related research}

In this section, we will discuss some previous literatures which have focus on developing a MAC protocol to guarantee a power consumption efficiency and/or delay optimization. In [11], an introduction to an efficient MAC protocol aimed to improve the performance of WBAN networks via providing an optimized power consumption and delay for MAC protocols by using low cost out of hand (on-demand) wakeup radio module to increase the sleeping time of the node. The waking-up process needs to be done through the WBAN coordinator which consumes the system resources.

Another scheme that aimed to reduce the collision ratio, low power consumption and high channel utilization in IEEE 802.15.6 is proposed in [12]. The main concept focus on dividing the contention access phase (CAP) in the new standard into sub-phases according to the delay requirements for four different categories of packets. The packets are categorized according to the packet priority into four levels such as highest prioirty medical services, general health service, mixed medical and non-medical service, and non-medical services. The proposed scheme shows an improvement over the IEEE 802.15.6 baseline and IEEE 802.15.4 in terms of collision ratio and average delay.

\section{The proposed dynamism for IEEE 802.15.6 Std}

The newly emerged IEEE 802.15.6 standard enabled three Physical (PHY) layer specifications, namely, NarrowBand (NB), Ultra WideBand (UWB) and Human Body Communication (HBC). With its two modes of operations, default and high QoS, UWB became a good candidate for supporting coexistence of Medical and consumer applications. On the Medium Access Control (MAC) layer, the IEEE 802.15.6 standard with beacon-enabled mode has defined an adjustable superframe structure that consists of contention-free and contention access periods as shown in Figure 1. The random access phase (RAP) would 


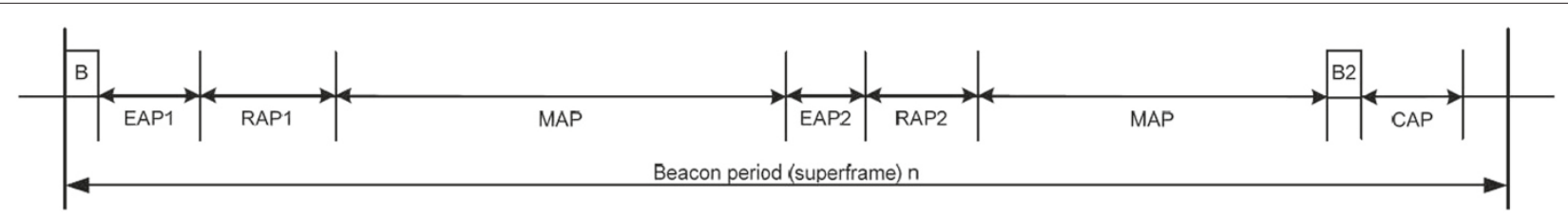

Figure 1 Superframe structure. Layout of access phases in a beacon period (superframe) for beacon mode.

be an efficient access phase for our proposal of spreading slotted Aloha. So, we assume the existence of RAP phase only during the numerical analysis of this paper.

Our previous proposal [6] introduced the spreading slotted Aloha to replace the conventional slotted Aloha random access scheme. Unlike the conventional slotted Aloha scheme, the spreading slotted Aloha does not assume that the (partial or full) collision occurs between two or more packets (coming from different orientation nodes) will destroy these packets. This is because these packets is spreaded with a unique pseudorandom code signature assigned to the users owning these packets. The uniqueness of these codes guarantee that only overlapped packets which spreaded with the same code will be destroyed.

Another important feature in the standard is the categorization of the contending nodes into eight different category according to these nodes' application priorities as shown in Table 1.

Obtaining a new contended allocation for the transmission or retransmission is conditioned with a contention probability $(\mathrm{CP})$ which should be more than or equal to a value $z$ that is uniformly drawn from $[0,1]$. A node with user priority $U P_{k}$ shall set its $C P$ initially to $\mathrm{CP}_{\max }$ if it did not obtain any contended allocation previously. After that, if the node did not grant access to contend the medium, it shall keep its CP as it is. In the other case, when the node granted access but failed to transmit the packet, the next $C P$ value will depend on the number of failures. If the number of failures is odd, CP keep its current value. If the number of failures is even, the $C P$ value should be

Table 1 User priorities and their corresponding contention probability

\begin{tabular}{lccc}
\hline User priority & Traffic designation & $\mathbf{C P}_{\max }$ & $\mathrm{CP}_{\text {min }}$ \\
\hline 0 (lowest) & Background (BK) & $\frac{1}{8}$ & $\frac{1}{16}$ \\
1 & Best effort (BE) & $\frac{1}{8}$ & $\frac{3}{32}$ \\
2 & Excellent effort (EE) & $\frac{1}{4}$ & $\frac{3}{32}$ \\
3 & Video (VI) & $\frac{1}{4}$ & $\frac{1}{8}$ \\
4 & Voice (VO) & $\frac{3}{8}$ & $\frac{1}{8}$ \\
5 & Medical data or network control & $\frac{3}{8}$ & $\frac{3}{16}$ \\
6 & High-priority medical data & $\frac{1}{2}$ & $\frac{3}{16}$ \\
7 (highest) & Emergency & 1 & $\frac{1}{4}$ \\
\hline
\end{tabular}

half the current value. If the halving makes the new $\mathrm{CP}$ less than $\mathrm{CP}_{\min }$, it should be set to $\mathrm{CP}_{\min }$. On the other side, if the nodes granted access for contention and successfully transmits the packet, it shall set its $\mathrm{CP}$ to $\mathrm{CP}_{\max }$. A flowchart for this mechanism is shown in Figure 2.

The dynamism of lowering the $\mathrm{CP}$ value by halving it every even number of failures gives a lower chance to transmit the packet successfully before it reaches the lowest $\mathrm{CP}\left(\mathrm{CP}_{\min }\right)$ which delays the packet transmission for a longer period because it has a lower chance to contend to the medium. In cases where the node has a long queue of data packets to transmit, the node's overall delay would increase.

In this sense, we propose a novel dynamism for lowering the $\mathrm{CP}$ value in the way to $\mathrm{CP}_{\min }$. In our proposal, halving the $\mathrm{CP}$ replaced with a reduction of the current $\mathrm{CP}$ as a ratio of the current queue length to the maximum allowable queue length. We denoted this ratio by $Q_{\text {thre }}$.

$$
\mathrm{Q}_{\text {thre }}=\frac{\text { Current queue length }}{\text { Maximum allowable queue length }}
$$

So in the case of an even number of transmission failure, the new lowered $\mathrm{CP}$ value will relay on how much

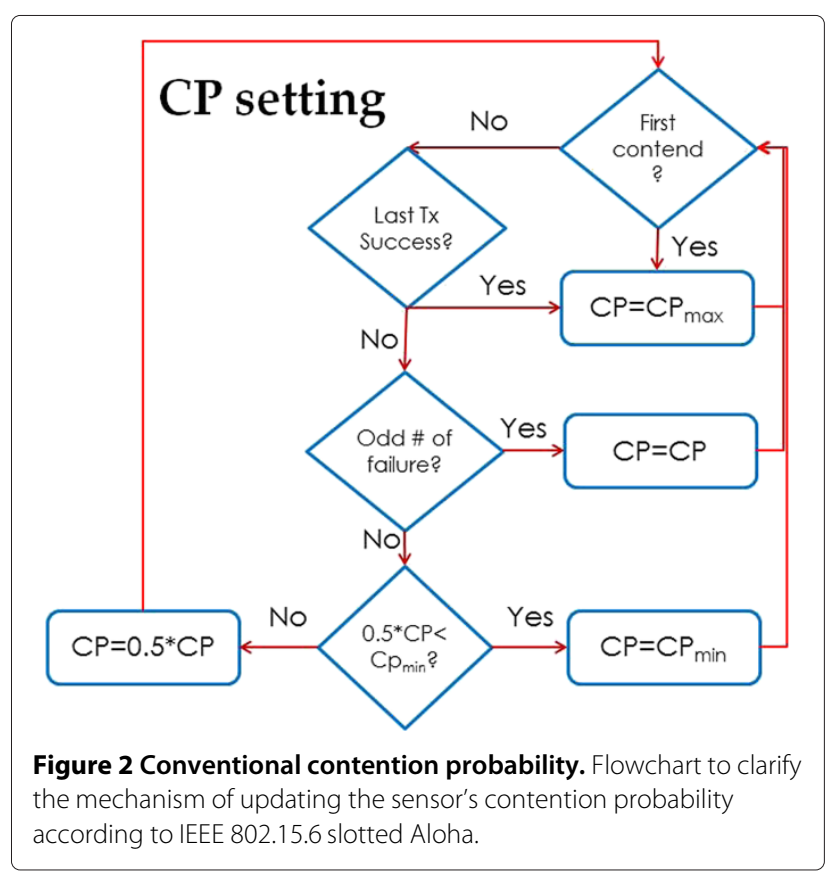


packets still remaining in that node's queue. If the node already transmit majority of its packets but fail to transmit the current packet, Qthre will be small which causes a fast lowering to $\mathrm{CP}$ value close to the original protocol settings. In case of a node with a near full queue, the bigger $\mathrm{Q}_{\text {thre }}$ value will cause a slow lowering to the $\mathrm{CP}$ value which allows a higher chance for the nodes to transmit their packets. A graphical representation for the proposed scheme is depicted in Figure 3.

\section{Performance evaluation}

In this section, we will evaluate the proposed dynamism numerically against the conventional dynamism using a MATLAB simulation built for a WBAN system and considering all the IEEE 802.15.6 standard parameters and specifications. First, we show the proposed scenario and related assumptions, then we show the numerical simulation results.

\subsection{System model}

The performance of the network has been investigated by simulating a WBAN consisting of a hub (coordinator) and a number of sensor nodes (end devices) employed for on-body to on-body communication network. The nodes belong to two different categories of priority and all nodes are organized in a one-hop star topology as shown in Figure 4. In addition, we assume that all transmitted data are generated at the sensor node then transmitted to the hub.

The first sensor category is employed for entertainment applications like video streaming which has a continuous traffic and can tolerate long latency with relaxed reliability requirements. According to the standard rules, these

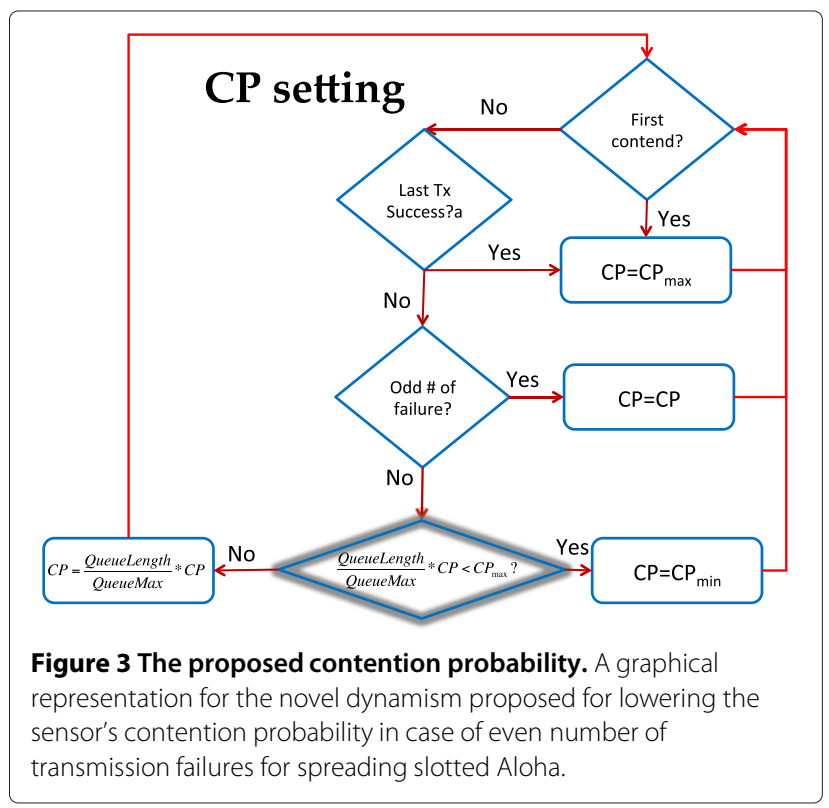

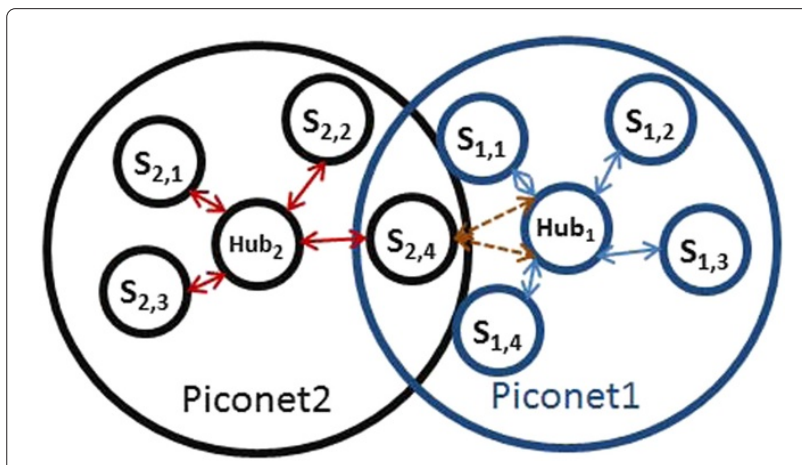

Figure 4 BAN to BAN interference. Interference between adjacent piconets.

non-medical nodes classified as low priority nodes and hence assigned a small contention probability starting from priority 0 and up to priority 3 as shown in Table 1 . In our simulations, we assumed a high information generation rate for these nodes, namely, 5,400, 7,000, 8,300 and 10,000 bps.

The second category is chosen to be employed for medical transmission; specifically, we used electroencephalography (EEG) sensors. These nodes are periodically transmitting low data rate but in emergency cases, it will have very critical medical information which requires instant and reliable delivery. According to the standard rules, the priorities assigned to these nodes vary from priority 4 and up to priority 7 according to Table 1 . In our simulations, we assumed that the EEG sensors uses information rates 2,000, 4,200, 4,400 and 4,800 bps.

Slotted Aloha implies a time slotted access structure in the RAP, where all slots are of equal sizes and determined by pAlohaSlotLength in the standard. All the simulations run for $1 \mathrm{~s}$.

Table 2 shows the simulation parameters used for the two categories. The common parameters follow closely the IEEE 802.15.6 [1] standard specifications for slotted Aloha random access scheme.

Table 2 Numerical simulation parameters

\begin{tabular}{lcc}
\hline Simulation parameter & Medical nodes & Non-medical nodes \\
\hline Information rates & $2,4.2,4.4,4.8 \mathrm{kbps}$ & $5.4,7,8.3,10 \mathrm{kbps}$ \\
Processing gain & 63 & 31 \\
Slot length & $10 \mathrm{~ms}$ & $10 \mathrm{~ms}$ \\
Symbol duration & $2.0513 \mu \mathrm{s}$ & $2.0513 \mu \mathrm{s}$ \\
SIFS & $75 \mu \mathrm{s}$ & $75 \mu \mathrm{s}$ \\
SYNC header duration & $40.3 \mu \mathrm{s}$ & $40.3 \mu \mathrm{s}$ \\
PHY header duration & $82.1 \mu \mathrm{s}$ & $82.1 \mu \mathrm{s}$ \\
MAC header duration & $148 \mu \mathrm{s}$ & $148 \mu \mathrm{s}$ \\
PHY data rate & $487 \mathrm{kbps}$ & $487 \mathrm{kbps}$ \\
\hline
\end{tabular}




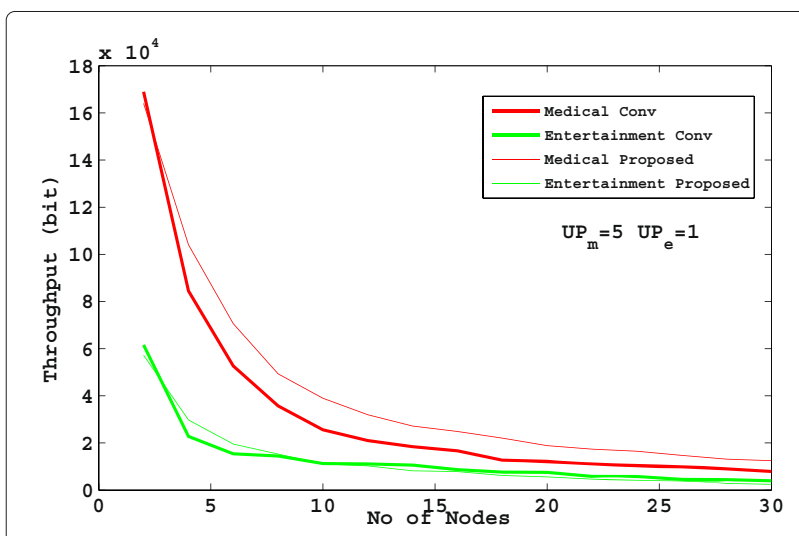

Figure 5 Throu5,1. Comparison between conventional and proposed dynamism in terms of throughput for priority 5 medical nodes and priority 1 entertainment nodes.

The proposed dynamism has been investigated in four cases; each contains two categories: category one contains medically oriented sensors and the category two contains non-medical oriented sensors. In addition to the IEEE 802.15.6 standard specifications which we follow closely, some assumptions are made for our simulation as follows:

- We assume a fixed slot length duration.

- Perfect synchronization between all the nodes is assumed so the cross correlation between the sensor nodes is almost zero because the spreading sequences is totally orthogonal.

- Due to perfect synchronisation assumption, there is no intra-BAN (within the same WBAN) cross correlation.

- Dropping the packets is due to the overflow of allowable retransmission which fixed to three and on failure to retrieve the transmitted packet.

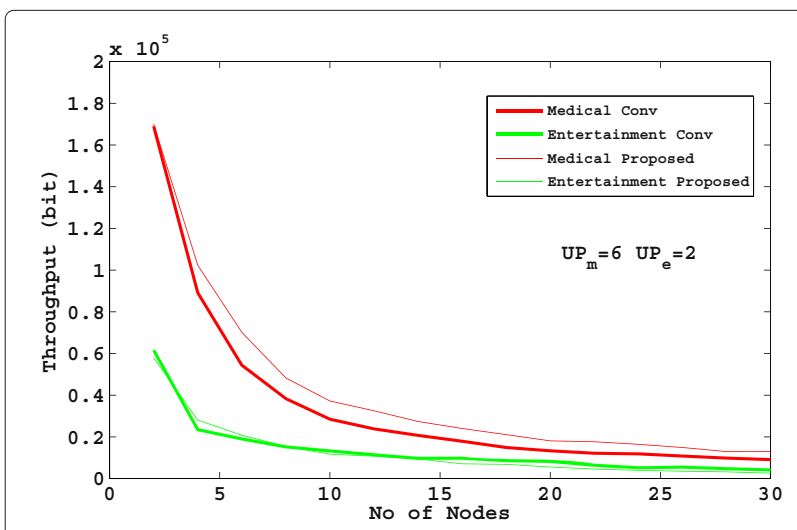

Figure 6 Throu6,2. Comparison between conventional and proposed dynamism in terms of throughput for priority 6 medical nodes and priority 2 entertainment nodes.

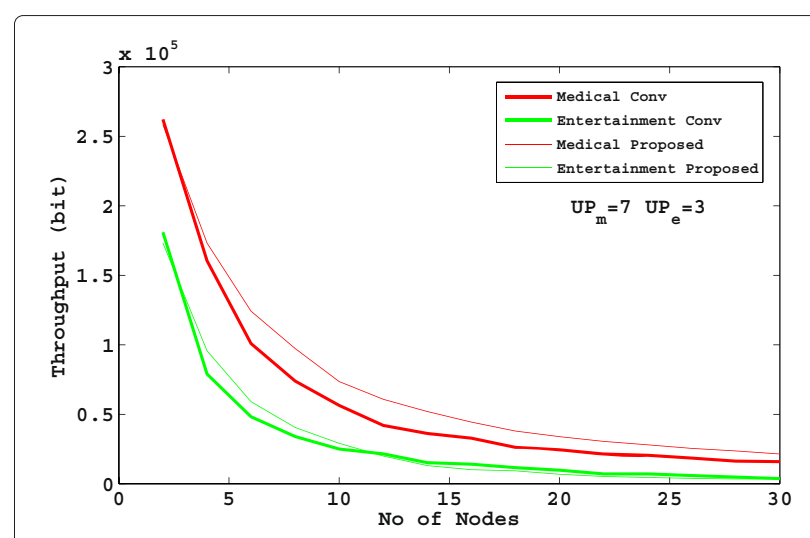

Figure 7 Throu7,3. Comparison between conventional and proposed dynamism in terms of throughput for priority 7 medical nodes and priority 3 entertainment nodes.

\subsection{Simulation results}

In this paper, the performance evaluation parameters are the average throughput, delay and the data packet dropping rate. Average throughput is defined as the number of correctly received bits divided by the total slots length (in seconds) which the node takes to transmit these bits correctly.

$$
S=\frac{\text { Number of correctly received bits }}{\text { Total consumed time }}
$$

The delay is calculated as the total time consumed in transmission during simulation time.

We evaluate the performance of the proposed dynamism via the numerical simulations assuming a fixed SNR value while variable number of nodes try to contend to the available time slot. The number of nodes trying to contend is equally distributed between medical-and nonmedical-oriented nodes. All the medical nodes assigned

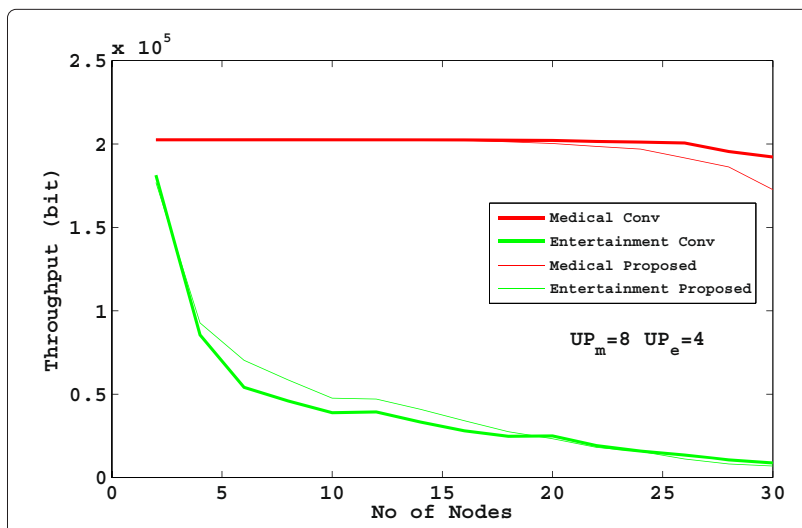

Figure 8 Throu8,4. Comparison between conventional and proposed dynamism in terms of throughput for priority 8 medical nodes and priority 4 entertainment nodes. 


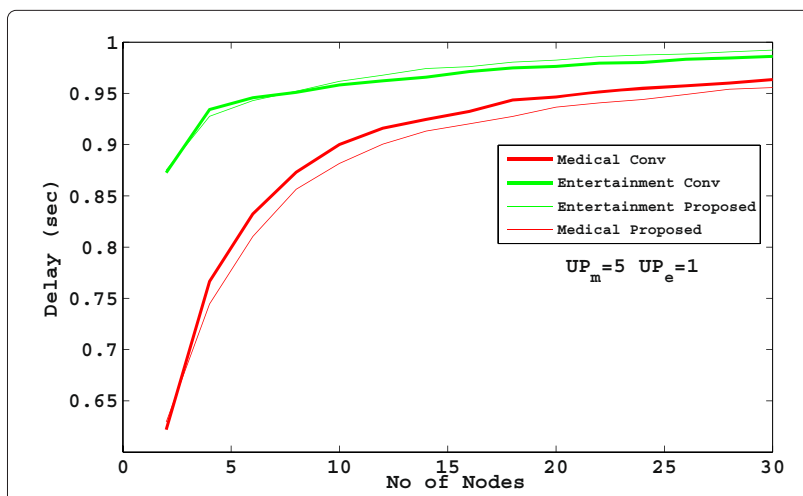

Figure 9 Delay5,1. Comparison between conventional and proposed dynamism in terms of delay for priority 5 medical nodes and priority 1 entertainment nodes.

the same spreading code length as shown in Table 2. The same way follows for the entertainment nodes.

Due to the higher contention probability that the proposed dynamism gives to the nodes with longer queues, the total number of correctly received data packets is higher. Therefore, the proposed dynamism's outperforms the conventional dynamism in many cases of the evaluation parameters.

A throughput comparison between the proposed and conventional CP dynamism for four different combinations of priority assignment is shown in Figures 5, 6, 7 and 8 for both medical and entertainment nodes. The figures show that there is a considerable improvement in the throughput when using the proposed dynamism especially for the medical sensors. When the number of medical/entertainment nodes are small, the proposed dynamism has almost the same results as the current dynamism in the IEEE 802.15.6 standard. Medical node throughput carried through an upswing for the proposed dynamism started when there are 4 contending nodes

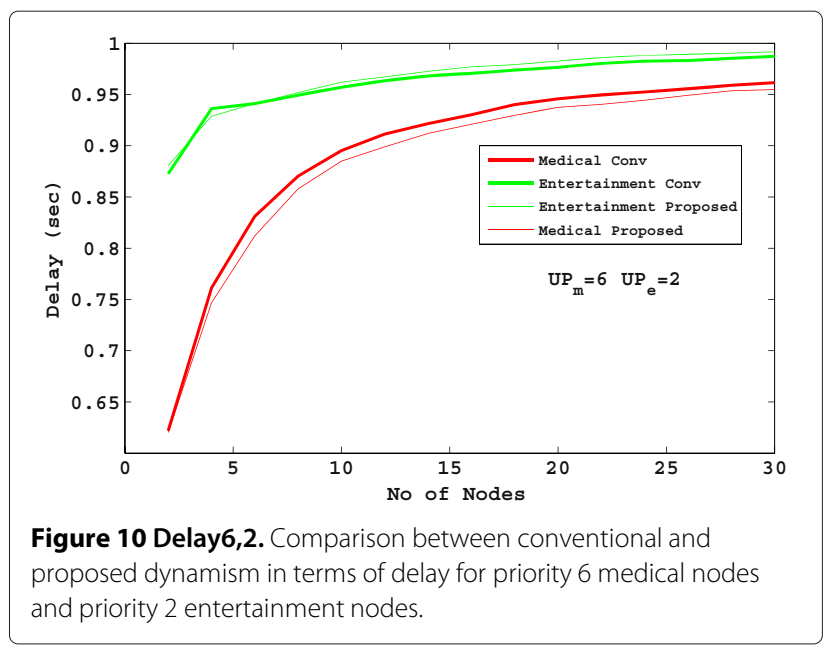

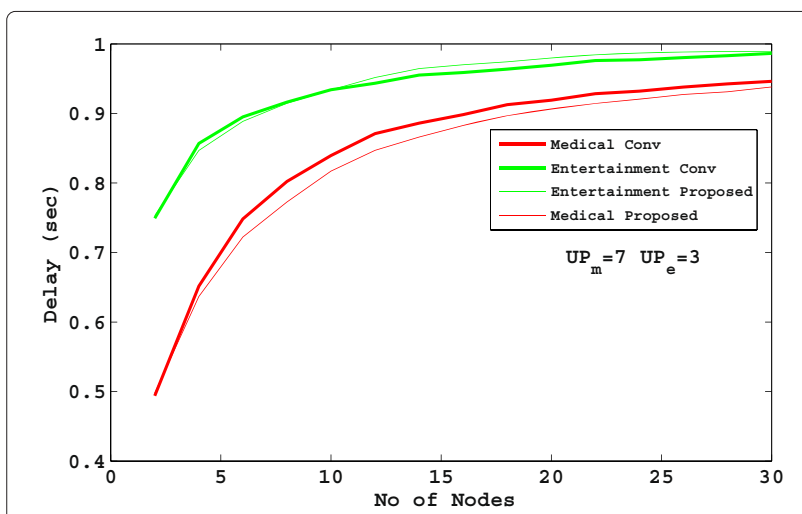

Figure 11 Delay7,3. Comparison between conventional and proposed dynamism in terms of delay for priority 7 medical nodes and priority 3 entertainment nodes.

in the system and continue up to 30 nodes. The best throughput performance achieved when 4 to 10 nodes is available.

As for the non-medical transmission sensors, an enhancement has been achieved but at a comparatively lower rate than its counterpart. The enhancement appears also when there are 4 to 8 nodes contending to the time slot. With the increment in the number of contending nodes, the proposed dynamism is very close to the conventional IEEE 802.15.6 dynamism.

For the delay, Figures 9, 10, 11, 12 depict the delay attitude as a comparison between proposed and conventional schemes. Close to the throughput attitude, a decrease in the delay particularly for medical sensors is achieved but it appears to be fixed all over the simulation.

Non-medical nodes have almost no improvement for the proposed dynamism over the conventional because the CP assigned to these nodes is already small and slowing down its shrinkage is not so effective.

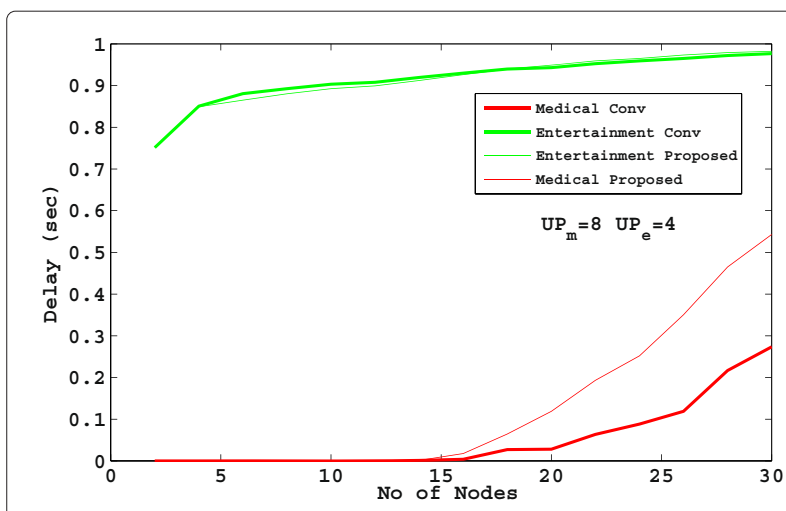

Figure 12 Delay8,4. Comparison between conventional and proposed dynamism in terms of delay for priority 8 medical nodes and priority 4 entertainment nodes. 


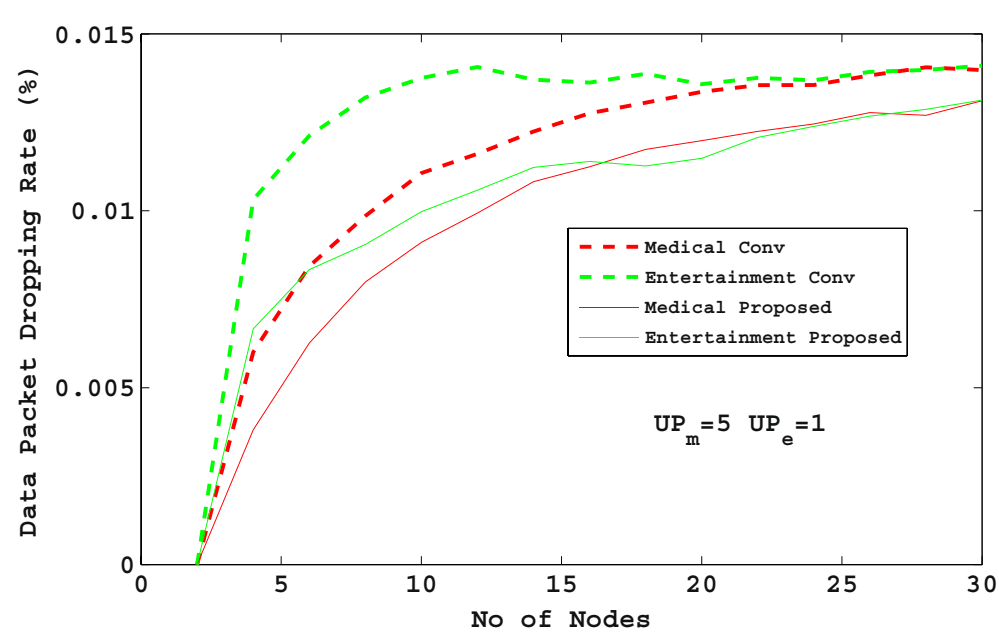

Figure 13 DropPkts5,1. Data packet dropping rate for priority 5 medical nodes and priority 1 entertainment nodes.

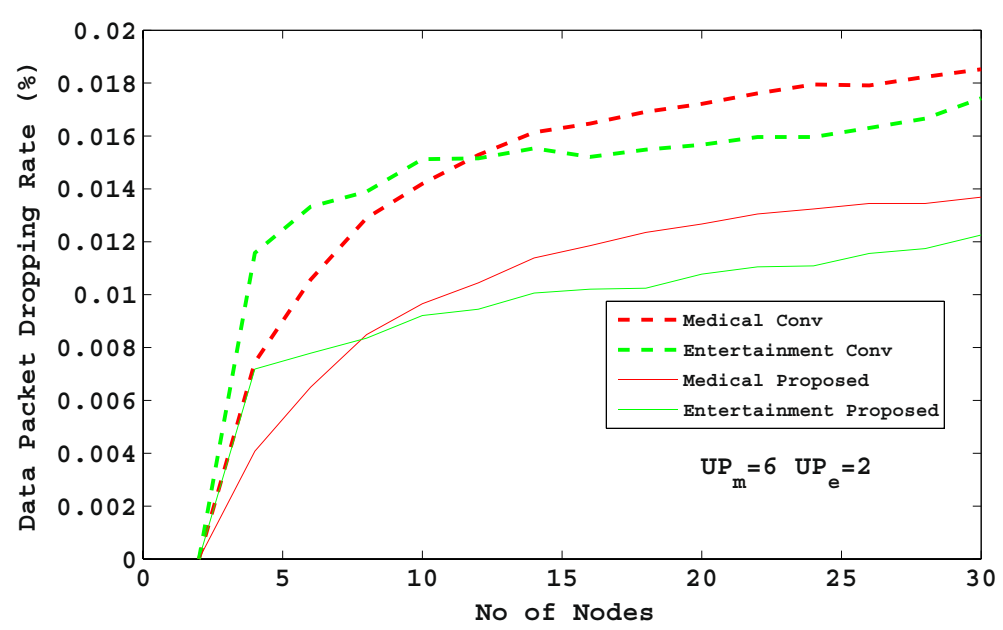

Figure 14 DropPkts6,2. Data packet dropping rate for priority 6 medical nodes and priority 2 entertainment nodes.

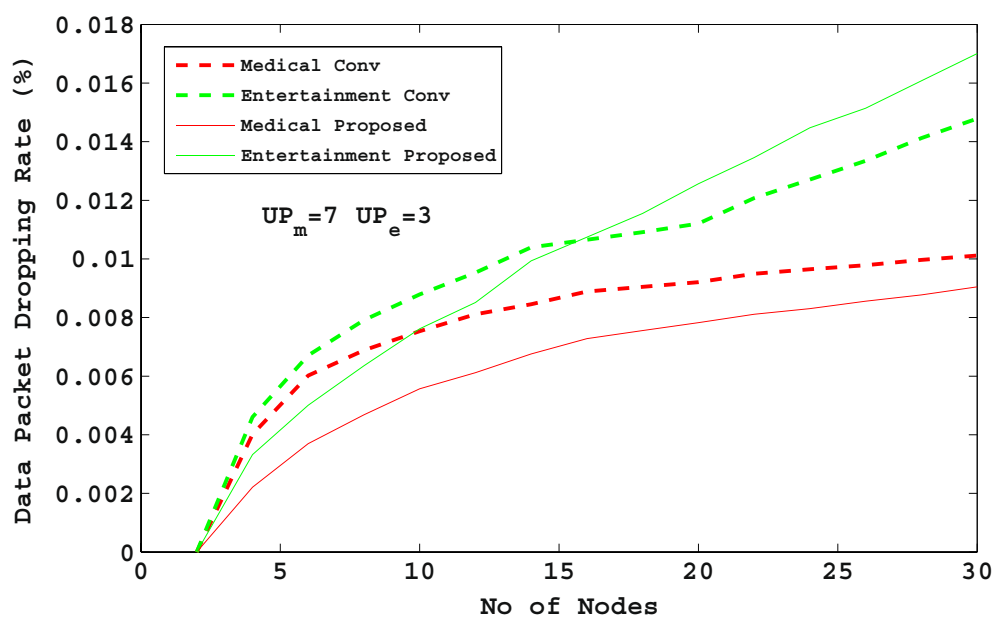

Figure 15 DropPkts7,3. Data packet dropping rate for priority 7 medical nodes and priority 3 entertainment nodes. 


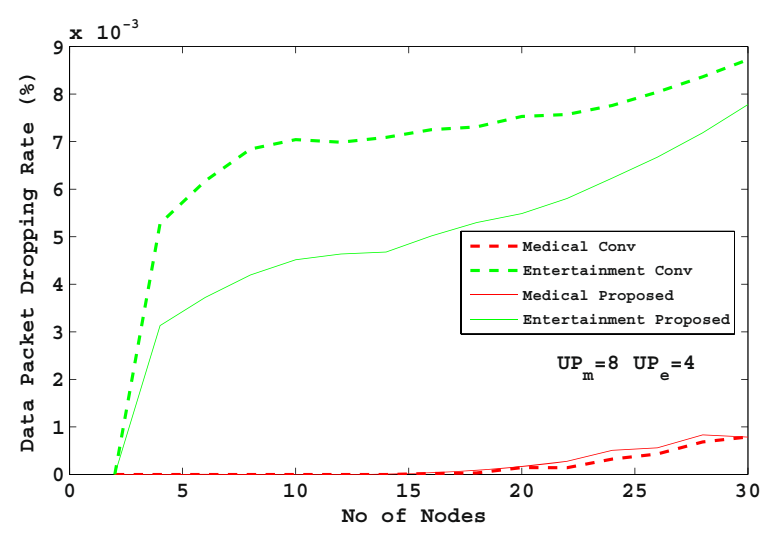

Figure 16 DropPkts8,4. Data packet dropping rate for priority 8 medical nodes and priority 4 entertainment nodes.

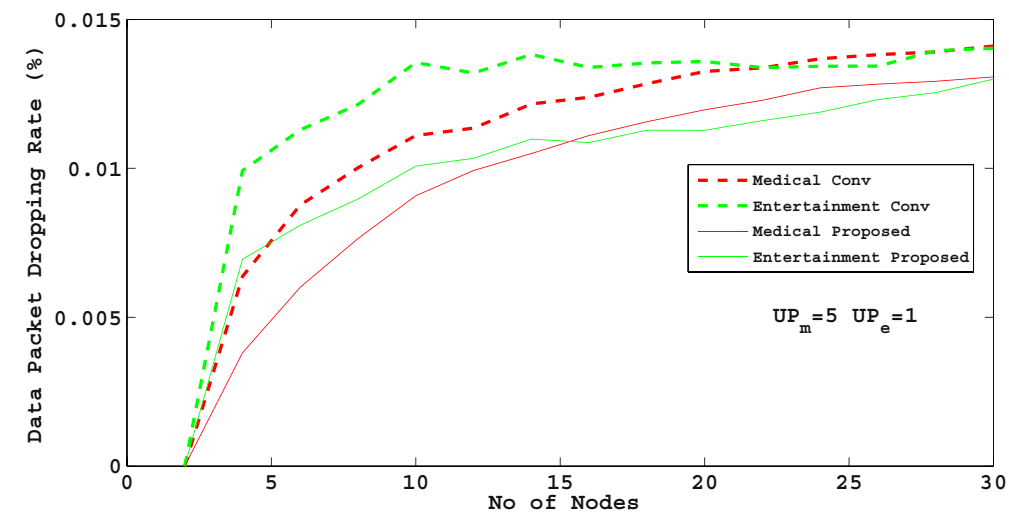

Figure 17 DropPktUP5,1VarSNR. Data packet dropping rate for priority 5 medical nodes and priority 1 entertainment nodes with a fluctuation of SNR during transmission.

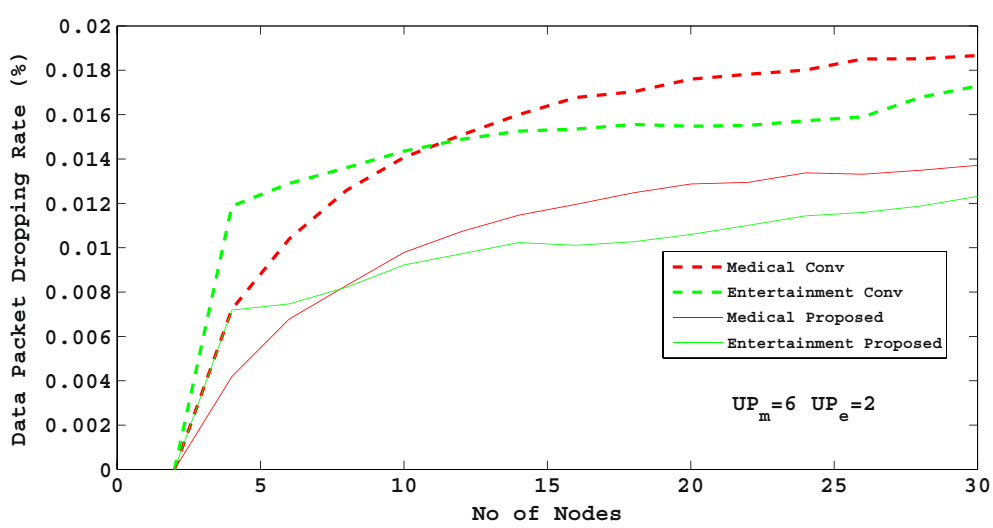

Figure 18 DropPktUP6,2VarSNR. Data packet dropping rate for priority 6 medical nodes and priority 2 entertainment nodes with a fluctuation of SNR during transmission. 


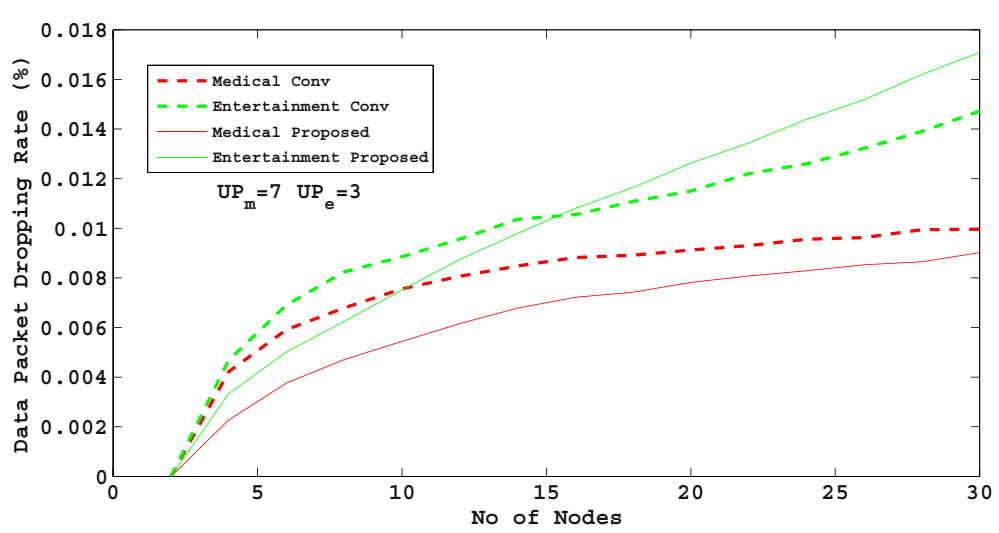

Figure 19 DropPktUP7,3VarSNR. Data packet dropping rate for priority 7 medical nodes and priority 3 entertainment nodes with a fluctuation of SNR during transmission.

The third evaluation parameter is the data packet dropping rate (in percentage) [13] which is defined as the ratio of dropped packets to the total correctly received packets. Dropped packets is the total number of the lost packets due to packet collision or retransmission overflow. The proposed dynamism gives more room for the users to transmit their packets before reaching the lowest contention probability value $\mathrm{CP}_{\min }$. We can see in the Figures 13, 14, 15 and 16 that the proposed dynamism offer a better performance than the conventional one.

Finally, our proposed scheme has been tested against SNR fluctuation during the different simulation trials in order to imitate a more realistic channel conditions where the noise power changing frequently which leads to a variation in the received power during the different trials. The proposed scheme depicted in Figures 17, 18, 19 and 20 reflect the stability of the proposed scheme under the frequent change in the SNR values which emphasizes on the preferability of the new dynamism compared to the conventional.

\section{Conclusion}

A proposal of a novel amendment on the current WBAN IEEE 802.15.6 standard has been discussed in this paper. The proposed dynamism is applied for spreading slotted Aloha of the newly emerged standard. The currently used contention probability CP aimed to tackle the problem of multi priority nodes in WBAN systems. In contrast to the currently used slotted Aloha scheme, the proposed dynamism allows slowly decrement of the $\mathrm{CP}$ value which provides higher chance to transmit the node's packet before reaching the smallest $C P$ value $C P_{\text {min }}$.

The numerical results depicted that the proposed dynamism outperforms the conventional in metrics of throughput, delay and data packet dropping rate. Moreover, it still giving a higher chance to the medical nodes

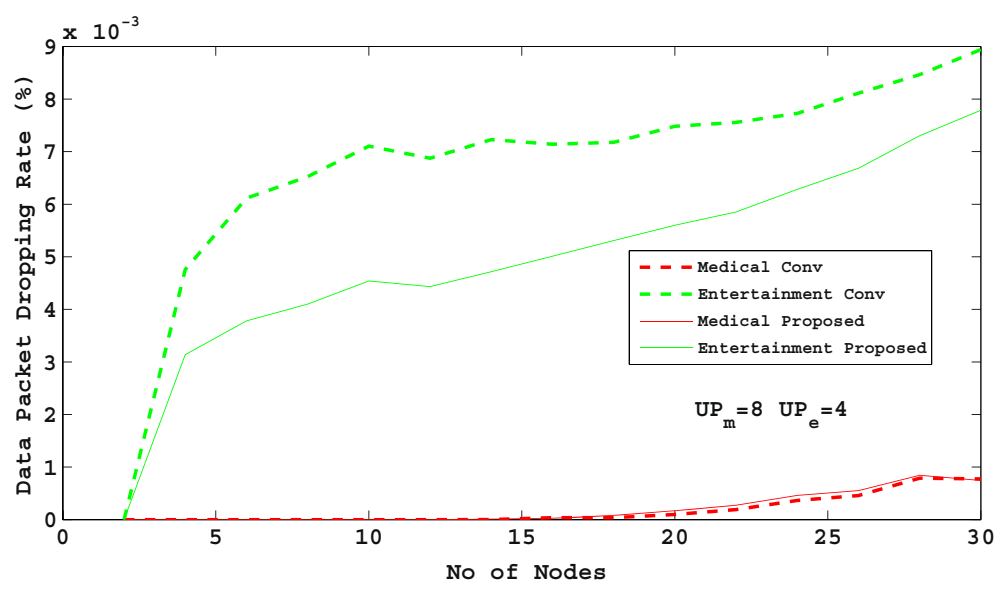

Figure 20 DropPktUP8,4VarSNR. Data packet dropping rate for priority 8 medical nodes and priority 4 entertainment nodes with a fluctuation of SNR during transmission. 
to transmit its packets first since it has a larger priority and near full queue as other nodes. When a medical

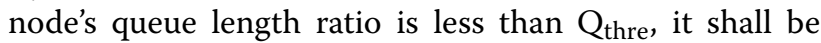
given comparatively less priority to transmit. Additionally, the proposed dynamism is stable against the change in the SNR value which occurred frequently during the transmission process.

\section{Competing interests}

The authors declare that they have no competing interests.

\section{Acknowledgements}

The first author would like to thank Dr. Jussi Haapola, Centre of Wireless Communications, University of Oulu, Finland and CWC-Nippon Co. Ltd. in Yokohama, Japan for his inspiration and guidance during this research.

Received: 29 January 2014 Accepted: 26 May 2014

Published: 11 June 2014

\section{References}

1. IEEE Standards Association, 802.15.6-2012 - IEEE Standard for Local and Metropolitan Area Networks - Part 15.6: Wireless Body Area Networks. (IEEE, Piscataway, 2012). doi:10.1109/IEEESTD.2012.6161600

2. K-S Kwak, S Ullah, N Ullah, An overview of IEEE 802.15.6 standard, in 2010 3 rd International Symposium on Applied Sciences in Biomedical and Communication Technologies (ISABEL), Rome, 7-10 Nov 2010, pp. 1-6

3. A Zhang, D Smith, D Miniutti, LW Hanlen, D Rodda, B Gilbert, Performance of Piconet Co-existence schemes in Wireless Body Area Networks, in IEEE Wireless Communications and Networking Conference (WCNC), Sydney 18-21 Apr 2010, pp. 1-6

4. B Zhen, H-B Li, R Kohno, Networking issues in medical implant communications. Int. J. Multi. Ubiq. Eng. 4(1) (2009)

5. N Abramson, THE ALOHA SYSTEM: another alternative for computer communications, in Proceedings of the November 17-19, 1970, Fall Joint Computer Conference (New York, NY, USA, 1970), pp. 281-285

6. M Fatehy, K Ryuji, Variable Spread Slotted Aloha Simulation Analysis over IEEE 802.15.6 using IR-UWB for Wireless Body Area Networks, in 7th European Modelling Symposium (EMS), Manchester, UK, 20-22 Nov 2013 pp. 572-577

7. D Makrakis, KMS Murthy, Spread slotted ALOHA techniques for mobile and personal satellite communication systems. IEEE J. Selected Areas Commun. 10(6), 985-1002 (1992)

8. OA Gonzalez, R Kohno, A spread slotted CDMA/ALOHA system with hybrid ARQ for satellite multiple access. IEEE J. Selected Areas Commun. 18(1), 123-131 (2000)

9. BH Jung, RU Akbar, DK Sung, Throughput, energy consumption, and energy efficiency of IEEE 802.15.6 body area network (BAN) MAC protocol, in 2012 IEEE 23rd International Symposium on Personal Indoor and Mobile Radio Communications (PIMRC), Sydney, 9-12 Sept 2012, pp. 584-589

10. S Ullah, M Chen, KS Kwak, Throughput and delay analysis of IEEE 802.15.6-based CSMA/CA protocol. J. Med. Syst. 36(6), 3875-3891 (2012)

11. MA Ameen, N Ullah, MS Chowdhury, SR Islam, K Kwak, A power efficient MAC protocol for wireless body area networks. EURASIP J. Wireless Commun. Netw. 2012(1), 33 (2012)

12. B Kim, J Cho, A novel priority-based channel access algorithm for contention-based MAC Protocol in WBANs, in Proceedings of the 6th International Conference on Ubiquitous Information Management and Communication, Kuala Lumpur, 20-22 Feb 2012 (ACM, New York, 2012), pp. 1:1-1:5

13. C Li, J Li, B Zhen, H-B Li, R Kohno, Hybrid unified-slot access protocol for wireless body area networks. Int. J. Wireless Inf. Netw. 17(3-4), 150-161 (2010)

doi:10.1186/1687-1499-2014-92

Cite this article as: Fatehy and Kohno: A novel contention probability dynamism for IEEE 802.15.6 standard. EURASIP Journal on Wireless Communications and Networking 2014 2014:92.

\section{Submit your manuscript to a SpringerOpen ${ }^{\mathcal{O}}$ journal and benefit from:}

- Convenient online submission

- Rigorous peer review

- Immediate publication on acceptance

- Open access: articles freely available online

- High visibility within the field

- Retaining the copyright to your article

Submit your next manuscript at $>$ springeropen.com 